\title{
THE HODGE FILTRATION AND CYCLIC HOMOLOGY
}

\author{
Charles Weibel
}

12 December, 1994

\begin{abstract}
We relate the "Hodge filtration" of the cohomology of a complex algebraic variety $X$ to the "Hodge decomposition" of its cyclic homology. If $X$ is smooth and projective, $H C_{n}^{(i)}(X)$ is the quotient of the Betti cohomology $H^{2 i-n}(X(\mathbb{C}) ; \mathbb{C})$ by the $(i+1)^{s t}$ piece of the Hodge filtration.
\end{abstract}

\section{Introduction}

The cyclic homology $H C_{*}(A)$ of an algebra $A$ has been intensely developed in the last decade. Recently this theory has been extended to schemes (in [WG] and $[\mathrm{W}]$ ), where something new happens: The cyclic homology of a smooth complex projective variety $X$, together with its $S$ maps, determines and is determined by the Hodge filtration of its classical cohomology.

Here is a more precise statement. If $X$ is a smooth projective variety, we prove that the quotient $H^{m} / F^{i+1} H^{m}$ of $H_{a n}^{m}(X, \mathbb{C})$ by the Hodge layer $F^{i+1} H_{a n}^{m}(X, \mathbb{C})$ is naturally isomorphic to the component $H C_{2 i-m}^{(i)}(X)$ of $H C_{2 i-m}(X)$; this isomorphism is compatible with the $S$ maps in cyclic homology, and with the isomorphisms $H_{a n}^{m}(X, \mathbb{C}) \cong H C_{2 i-m}^{(i)}(X)$ for $i \geq m$. For this reason, I call the underlying decomposition $H C_{n}(X)=\prod H C_{n}^{(i)}(X)$ the Hodge decomposition of cyclic homology; it is also called the $\lambda$-decomposition $[\mathrm{FT}][\mathrm{L}]$.

The above result was announced in [W, 9.8.19], and forms the core $(\S 4)$ of the present paper.

The Hodge filtration of $H_{a n}^{m}(X, \mathbb{C})$ is described by the rows of the "Hodge diamond" associated to a smooth projective variety $X$. The columns of the Hodge diamond have a similar interpretation; they describe the "Hodge-type" decomposition $H H_{n}(X)=\prod H H_{n}^{(i)}(X)$ of Hochschild homology [GS].

We also give some localization sequences for cyclic homology. As an application, consider the coordinate ring $A$ of a smooth affine curve over $k$. If the curve has genus $g$ and $n$ rational points at infinity, we show that $H C_{1}(A)=k^{2 g+n-1}$.

Here is an outline of this paper. The Hochschild result is proven in $\S 1$ and revisited in $\S 4$. The cyclic homology of mixed complexes of sheaves is developed in $\S 2$. An important example is the mixed complex $\left(\Omega_{X}^{*}, 0, d\right)$; its periodic cyclic homology is a product of copies of the classical de Rham cohomology $H_{d R}^{*}(X / k)$ of $X$ over $k$ (see $[\mathrm{GdR}])$. This will be our segue into our main result, since when $X$

Author partially supported by NSF grants 1991-1994, as well as by CNRS funds during the academic year 1992-1993 
is smooth the cyclic homology of $\left(\Omega_{X}^{*}, 0, d\right)$ will agree with the cyclic homology of $X$, and it is well-known $[\mathrm{GdR}]$ that $H_{d R}^{*}(X / \mathbb{C}) \cong H_{a n}^{*}(X, \mathbb{C})$; Of course, if $X$ is singular then the calculations of $[\mathrm{BH}]$ show that $H_{d R}^{*}(X / \mathbb{C})$ does not always equal $H_{a n}^{*}(X, \mathbb{C})$.

The decomposition of cyclic homology is developed in $\S 3$. The most important result (3.4) is an extension of a theorem of Feigin and Tsygan [FT]: for every quasiprojective variety $X$ over $\mathbb{C}$ the analytic cohomology $H_{a n}^{m}(X, \mathbb{C})$ is isomorphic to the component $H P_{2 i-n}^{(i)}(X)$ of periodic cyclic homology. We also give some localization sequences (3.7) which allow us to better describe the cyclic homology of affine curves (see 3.6 and 5.5).

The last section ( $\S 5$ ) is devoted to an analysis of the cyclic homology of singular varieties. We show that cyclic homology defines a natural filtration $G^{i} H^{m}$ on $H^{m}(X, \mathbb{C})$, which is contained in - but in general smaller than - the Hodge filtration of Deligne. It suggests that the cyclic homology of a smooth hypercover of $X$, which is directly related to Deligne's Hodge filtration, is just as useful as the cyclic homology of $X$. Unfortunately there seems to be no way to translate this idea into the universe of noncommutative algebras.

Notation. We will fix a commutative ring $k$ containing $\mathbb{Q}$, and consider quasicompact quasi-separated schemes $X$ over $k$; the reader may restrict to noetherian schemes over a field of characteristic zero without much loss of generality. (All noetherian schemes are quasi-compact and separated by [EGA, $\left.0_{I V}(1.2 .8)\right]$.)

We will write $\Omega_{X}$ for the sheaf $\Omega_{X / k}$ of relative Kähler differentials on $X$. 'Tot' means the product total complex. We will freely identify chain complexes $C_{*}$ with cochain complexes $C^{*}$ via $C_{n}=C^{-n}$. The translation $C[p]$ of $C$ is indexed as in $[\mathrm{W}, 1.2 .8]: C[p]_{n}=C_{n+p}, C[p]^{n}=C^{n-p}$.

We will freely refer to the "Cartan-Eilenberg" hypercohomology $\mathbb{H}^{*}\left(X, \mathcal{C}^{*}\right)$ of a cochain complex $\mathcal{C}^{*}$ of sheaves (say of $k$-modules) which is not bounded below. This is the hypercohomology used in [T], [WG] and [W1], and it differs slightly from the derived category hypercohomology of [Sp]. (See the appendix to [W1] for a discussion.) To define it, choose an injective Cartan-Eilenberg resolution $\mathcal{C}^{*} \rightarrow I^{* *}$; its global sections $\Gamma I^{* *}$ form a double complex of injective $k$-modules. The hypercohomology complex $\mathbb{H}\left(X, \mathcal{C}^{*}\right)$ is just the total complex $\operatorname{Tot} \Gamma I^{* *}$, and the hypercohomology of $\mathcal{C}^{*}$ is simply its cohomology: $\mathbb{H}^{n}(X, \mathcal{C})=H^{n} \mathbb{H}(X, \mathcal{C})$.

\section{$\S 1$. Hochschild homology}

We begin with a discussion of the Hodge decomposition for Hochschild homology, where things are less intricate.

Let $X$ be a quasi-compact quasi-separated scheme over a fixed commutative ring $k$. To define the Hochschild homology of $X$, we may sheafify the usual Hochschild complex $C_{*}(A)=A^{\otimes *+1}$ in the Zariski topology to obtain a chain complex $\mathcal{C}_{*}$ of sheaves.

$$
\mathcal{C}_{*}: \quad \cdots \rightarrow \mathcal{O}_{X}^{\otimes *+1} \rightarrow \cdots \rightarrow \mathcal{O}_{X} \otimes_{k} \mathcal{O}_{X} \rightarrow \mathcal{O}_{X} \rightarrow 0
$$

As in [WG] [W1], the Hochschild homology of $X$ over $k$ is defined as the hypercohomology of this complex, reindexed as a cochain complex by $\mathcal{C}^{n}=\mathcal{C}_{-n}$ : 
For an affine scheme $X=\operatorname{Spec} A$, this $H H_{n}(X)$ agrees with the classical group $H H_{n}(A)$ by [WG, 4.1]. Although $X$ can have negative Hochschild homology - see (1.1) below - we will see in 1.2 that it is bounded below. For example, when $X$ is noetherian over $k$ then $H H_{n}(X)=0$ for $n<-\operatorname{dim}(X)$ [WG, 4.3].

We now assume that $k$ contains $\mathbb{Q}$. In this case, for each commutative algebra $A$ there is a natural decomposition of $C_{*}(A)$ as a product (or direct sum) of chain subcomplexes $\mathcal{C}_{*}^{(i)}(A), i \geq 0$.

$$
C_{*}(A)=A \times C_{*}^{(1)}(A) \times C_{*}^{(2)} \times \cdots \times C_{*}^{(i)}(A) \times \cdots \quad \text { with } C_{n}^{(i)}(A)=0 \text { if } i>n .
$$

This gives $H H_{n}(A)=\prod_{i=0}^{n} H H_{n}^{(i)}(A)$, where $H H_{n}^{(i)}(A)=H_{n} C_{*}^{(i)}(A)$. Following [GS], we shall call this the Hodge decomposition; it is called the $\lambda$-decomposition in $[\mathrm{L}]$.

For schemes we set $H H_{n}^{(i)}(X)=\mathbb{H}^{-n}\left(X, \mathcal{C}_{*}^{(i)}\right)$; we will see in 1.3 below that for any affine scheme $X=\operatorname{Spec}(A)$ we have $H H_{n}^{(i)}(X)=H H_{n}^{(i)}(A)$. When $i=0$, the hypercohomology of $\mathcal{C}_{*}^{(0)}=\mathcal{O}_{X}$ is just cohomology:

$$
H H_{n}^{(0)}(X)= \begin{cases}H^{0}\left(X, \mathcal{O}_{X}\right) & \text { if } n=0 \\ H^{-n}\left(X, \mathcal{O}_{X}\right) & \text { if } n \text { is negative } \\ 0 & \text { if } n \text { is positive. }\end{cases}
$$

We will prove in 1.3 that $H H_{n}(X)$ is the product of the $H H_{n}^{(i)}(X)$. In order to do so, we introduce the sheafification $\mathcal{H H}_{n}^{(i)}$ of the presheaf $H H_{n}^{(i)}$. These sheaves are summands of $\mathcal{H} \mathcal{H}_{n}$, so they are quasi-coherent by [WG, 0.4]. Moreover $\mathcal{H} \mathcal{H}_{n}^{(i)}=0$ for $n<i$, and $\mathcal{H} \mathcal{H}_{n}^{(n)} \cong \Omega_{X}^{n}$.

Proposition 1.2. There is a fourth quadrant spectral sequence for each $i$, which is bounded and converges if $X$ is quasi-compact and quasi-separated:

$$
E_{2}^{p q}=H^{p}\left(X, \mathcal{H} \mathcal{H}_{-q}^{(i)}\right) \Rightarrow H H_{-p-q}^{(i)}(X)
$$

Moreover, if $X$ is noetherian then $H H_{n}^{(i)}(X)=0$ if $i>n+\operatorname{dim}(X)$.

Proof. (Cf. [WG, 4.2].) This is just the hypercohomology spectral sequence [EGA, $\left.0_{I I I}(12.4)\right][\mathrm{T}, 1.46]$ associated to the complex $\mathcal{C}_{*}^{(i)}$. When $X$ is quasi-compact and quasi-separated, there is a uniform bound $d$ on the cohomology of quasi-coherent sheaves. (See [EGA, III(1.4.12) and IV(1.7.21)].) Since $E_{2}^{p q}=0$ if $p>d$ or $q>-i$, we have $H H_{n}^{(i)}(X)=0$ if $n>d-i$.

Proposition 1.3. When $X$ is quasi-compact and quasi-separated, there is a finite decomposition for each $n$ :

$$
H H_{n}(X)=H H_{n}^{(0)}(X) \times H H_{n}^{(1)}(X) \times \cdots \times H H_{n}^{(i)}(X) \times \cdots
$$

We call this the Hodge decomposition of $H H_{n}(X)$. When $X=\operatorname{Spec}(A)$ we have $H H_{n}^{(i)}(X)=H H_{n}^{(i)}(A)$, and the Hodge decompositions of $H H_{n}(X)$ and $H H_{n}(A)$ 
Proof. We have to show that the natural map $p_{X}: H H_{n}(X) \rightarrow \prod H H_{n}^{(i)}(X)$ is an isomorphism, since only finitely many of the $H H_{n}^{(i)}(X)$ in the product are nonzero for each $n$ by 1.2. For an affine scheme $X=\operatorname{Spec} A$, we argue as in [WG, 4.1]; the spectral sequence collapses along the line $p=0$ to yield $H H_{n}^{(i)}(X)=H H_{n}^{(i)}(A)$, and we recover the usual Hodge decomposition of $H H_{n}(A)$. We now proceed as in [W1, 0.5]. If $X$ is separated, the result follows from induction on the size of an affine cover, the Mayer-Vietoris sequence and the 5-lemma. Another induction, on the size of a quasi-affine cover, proves the result for all quasi-separated $X$.

The following two results follow from the hypercohomology spectral sequence.

Corollary 1.4. (announced in [W, 9.8.19]) If $X$ is smooth over $k$, then for all $i$ and $n$

$$
H H_{n}^{(i)}(X)=H^{i-n}\left(X, \Omega_{X}^{i}\right)
$$

Proof. If $X$ is smooth over $k$ then $\mathcal{H H}_{n}^{(i)}=0$ unless $n=i$. Therefore the spectral sequence 1.2 degenerates along the row $q=-i$.

Corollary 1.5. (Cf. [V, 4.2]) If $X$ is locally a complete intersection, then

$$
H H_{n}^{(i)}(X)=0 \quad \text { whenever } i<n / 2 \text {. }
$$

Proof. If $A$ is locally a complete intersection over $k$, the cotangent complex $\mathbb{L}_{A / k}$ has projective dimension $\leq 1$ by $[\mathrm{Q}, 5.4]$. As in [Q], this means that the AndréQuillen homology $D_{n}(A / k)$ vanishes for $n>1$, and the variant groups $D_{n}^{(i)}(A / k)=$ $H_{n}\left(\Lambda^{i} \mathbb{L}_{A / k}\right)$ vanish whenever $n>i$. Using the dictionary (see [Ro]) that $H H_{n}^{(i)}(A)$ equals $D_{n-i}^{(i)}(A / k)$, this means that $\mathcal{H H}_{n}^{(i)}=0$ whenever $n>2 i$. Hence the spectral sequence 1.2 is zero below the row $q=-2 i$, and we may read the result off from this.

Remark. It is suggestive to write $D_{n}(X / k)$ for $H H_{n+1}^{(1)}(X)$, since this is a natural extension of André-Quillen homology to schemes over $k$.

Example 1.6. Let $X$ be a reduced (singular) curve over a perfect field $k$. Then $\mathcal{H} \mathcal{H}_{0}^{(0)}=\mathcal{O}_{X}$ and $\mathcal{H} \mathcal{H}_{1}^{(1)}=\Omega_{X}$; all the other sheaves $\mathcal{H} \mathcal{H}_{n}^{(i)}$ are skyscraper sheaves supported on the (finite) singular locus. So the spectral sequence 1.2 degenerates to yield $H H_{-1}(X)=H H_{-1}^{(0)}(X)=H^{1}\left(X, \mathcal{O}_{X}\right), H H_{0}(X)=H H_{0}^{(0)}(X) \oplus H H_{0}^{(1)}(X)$,

$$
H H_{0}^{(0)}(X)=H^{0}\left(X, \mathcal{O}_{X}\right) \text { and } H H_{0}^{(1)}(X)=H^{1}\left(X, \Omega_{X}\right),
$$

$H H_{n}^{(i)}(X)=H^{0}\left(X, \mathcal{H} \mathcal{H}_{n}^{(i)}\right)$ for $n \geq 1$. In particular, $H H_{n}^{(i)}(X)=0$ if $i>n>0$ and

$$
H H_{1}(X)=H H_{1}^{(1)}(X)=H^{0}\left(X, \Omega_{X}\right)
$$

\section{$\S 2$. Mixed complexes of sheaves}

For purposes of studying the Hodge decomposition of cyclic homology, it is useful to introduce the notion of cyclic hyperhomology for mixed complexes $(\mathcal{M}, b, B)$ of sheaves on a site $X$. By a mixed complex of sheaves we mean a nonnegative chain complex $\mathcal{M}_{*}$ of sheaves with differential $b$, together with a family of maps 
Definition 2.0. Let $(\mathcal{M}, b, B)$ be a mixed complex of sheaves on $X$. The Hochschild hyperhomology of $\mathcal{M}$ is $H H_{n}(\mathcal{M})=\mathbb{H}^{-n}(X,(\mathcal{M}, b))$. The cyclic hyperhomology of $\mathcal{M}$ is defined to be $H C_{n}(\mathcal{M})=\mathbb{H}^{-n}\left(X\right.$, Tot $\left.\mathcal{B}_{* *}(\mathcal{M})\right)$, where $\mathcal{B}_{* *}(\mathcal{M})$ is Connes' $(b, B)$ double complex (of sheaves) for $\mathcal{M}$, as in [L, 2.5.13] or [W, 9.8.2].

The usual homological yoga yields "SBI" sequences (2.1.3) connecting $H H_{*}(\mathcal{M})$ and $H C_{*}(\mathcal{M})$, because hypercohomology is a cohomological functor. (See the appendix to [W1], [T, 1.35] or [WG, A.4].) In addition, the column filtration on $\mathcal{B}_{* *}$ gives rise to a generalization of Connes' spectral sequence: $E_{p q}^{1}=H H_{q-p}(\mathcal{M}) \Rightarrow$ $H C_{p+q}(\mathcal{M})$, which converges if $X$ has finite cohomological dimension by [D, 1.4].

2.1. Because inverse limits of sheaves are poorly behaved, we need care in defining periodic and negative cyclic hyperhomology. Write $\mathbb{H}$ for the hypercohomology complex $\mathbb{H}\left(X\right.$, Tot $\left.\mathcal{B}_{* *}(\mathcal{M})\right)$, so that by definition $H C_{n}(\mathcal{M})$ is $H^{-n}(\mathbb{H})$. We may arrange that the map $\mathbb{H}[2] \rightarrow \mathbb{H}$ induced by the usual map $S: \mathcal{B}_{* *}[2] \rightarrow \mathcal{B}_{* *}$ is onto. This allows us to form both a surjective tower of complexes of sheaves $\left\{\operatorname{Tot} \mathcal{B}_{* *}[2 p]\right\}$ and a surjective tower of complexes of injective $k$-modules

$$
\cdots \stackrel{S}{\longrightarrow} \mathbb{H}[2 p] \stackrel{S}{\longrightarrow} \mathbb{H}[2 p-2] \stackrel{S}{\longrightarrow} \cdots \stackrel{S}{\longrightarrow} \mathbb{H}
$$

Write $\operatorname{holim}_{p} \mathbb{H}[2 p]$ for the inverse limit of the tower (2.1.1); this chain complex is quasi-isomorphic to the usual homotopy limit by [BK, XI.4.1], [T, 5.6] or [BN, 2.3.1]. We define the periodic cyclic hyperhomology of $\mathcal{M}$ to be $H P_{n}(\mathcal{M})=$ $H^{-n} \operatorname{holim}_{p} \mathbb{H}[2 p]$. It is related to cyclic homology by the usual Milnor sequence for the tower (2.1.1); see [W, 3.5.8], [BK, IX.3.1] or [T, 5.41].

Milnor sequence 2.1.2. For any $X$ and $\mathcal{M}$ we have an exact sequence

$$
0 \rightarrow \varliminf^{1}{ }^{1} H C_{n+2 i+1}(\mathcal{M}) \rightarrow H P_{n}(\mathcal{M}) \rightarrow \varliminf_{\lim } H C_{n+2 i}(\mathcal{M}) \rightarrow 0 .
$$

The negative cyclic hyperhomology $H N_{*}(\mathcal{M})$ of $\mathcal{M}$ is defined as the (co)homology of the kernel of the natural surjection holim $_{p} \mathbb{H}[2 p] \rightarrow \mathbb{H}[-2]$; The resulting long exact hypercohomology sequence is compatible with the usual "SBI" sequence:

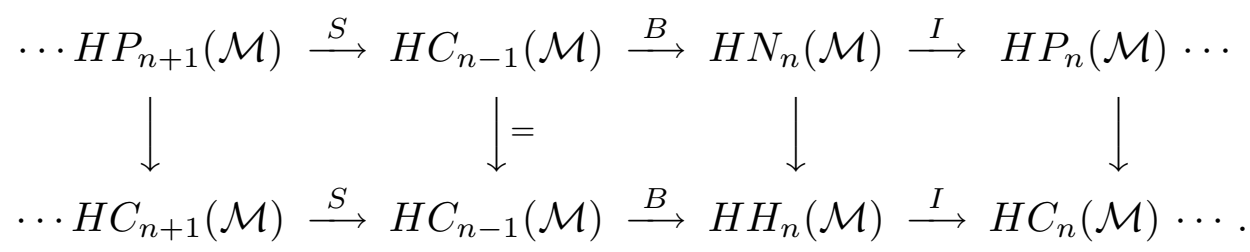

Main Example 2.2 (HC of a scheme). Let $X$ be a scheme over $k$. In $\S 1$ we introduced the chain complex $\mathcal{C}_{*}=\mathcal{O}_{X}^{\otimes *+1}$ and wrote $H H_{n}(X)$ for $H H_{n}\left(\mathcal{C}_{*}\right)$. In fact, $\mathcal{C}_{*}$ forms a mixed complex; the map $B$ is the sheafification of the usual operator $B$ in cyclic homology. We shall write $H C_{*}(X)$ for $H C_{n}\left(\mathcal{C}_{*}\right)$, as was done in [WG] and [W1]. We shall also write $H P_{*}(X)$ for $H P_{n}\left(\mathcal{C}_{*}\right)$ and $H N_{*}(X)$ for $H N_{n}\left(\mathcal{C}_{*}\right)$.

If $X=\operatorname{Spec}(A)$ is affine then $H C_{*}(X)=H C_{*}(A)$ by [W1]. By (2.1.2) we also have $H P_{*}(X)=H P_{*}(A)$ and therefore also $H N(X)=H N_{*}(A)$. 
Example 2.3 (Sheaves). At the other extreme, we may regard any sheaf $\mathcal{M}$ as a mixed complex, concentrated in degree 0. Clearly $H H_{n}(\mathcal{M})=H^{-n}(X, \mathcal{M})$. In this case $\operatorname{Tot} \mathcal{B}_{* *}=\prod_{i \geq 0} \mathcal{M}[-2 i]$, so $H C_{n}(\mathcal{M})=\prod_{i \geq 0} H^{2 i-n}(X, \mathcal{M})$. (See [W1, A.4].) Also for all $n \geq 0$ the Milnor sequence 2.1.2 yields $H P_{n}(\mathcal{M})=H C_{n}(\mathcal{M})$.

Another consequence of our homological yoga is this. A short exact sequence of mixed complexes gives rise to short exact sequences of double complexes $\mathcal{B}_{* *}$, and hence to long exact sequences for $H H_{*}, H C_{*}, H P_{*}$ and $H N_{*}$. We also have the following general principle.

Lemma 2.5. 1) If $(\mathcal{C}, b, B)$ is a mixed complex whose underlying chain complex of sheaves $(\mathcal{C}, b)$ is acyclic, then

$$
H H_{n}(\mathcal{C})=H C_{n}(\mathcal{C})=H P_{n}(\mathcal{C})=H N_{n}(\mathcal{C})=0 \quad \text { for all } n .
$$

2) Let $f: \mathcal{L} \rightarrow \mathcal{M}$ be a morphism of mixed complexes of sheaves on $X$. If $f$ is a quasi-isomorphism on the underlying chain complexes of sheaves, then $f$ induces an isomorphism on $H H_{*}, H C_{*}, H P_{*}$ and $H N_{*}$.

Proof. 1) The double complex $\mathcal{B}_{* *}$ associated to the mixed complex $\mathcal{C}$ has acyclic columns, and this implies (see [W, 2.7.3]) that the total complexes of sheaves is acyclic. By the hypercohomology spectral sequence, the hypercohomology of these acylic complexes vanishes. This gives vanishing of $H_{*}$ and $H C_{*}$; the vanishing of $H P_{*}$ and $H N_{*}$ is a formal consequence. Part 2) follows, since the mapping cone $\mathcal{C}$ of $f$ is a mixed complex, acyclic as a cochain complex.

Example 2.6. (Normalized mixed complex) Let $\overline{\mathcal{C}}_{*}$ be the normalized chain complex $\mathcal{O}_{X} \otimes \overline{\mathcal{O}}_{X}^{\otimes n}$ associated to the complex $\mathcal{C}_{*}$ of 2.2 ; it too is a mixed complex and is quasi-isomorphic to $\mathcal{C}_{*}$ [L, 1.1.14] [W, 9.8.4]. Lemma 2.5 assures us that we could have defined cyclic homology using $\overline{\mathcal{C}}_{*}$ instead of $\mathcal{C}_{*}$.

Example 2.7. (de Rham theory) Setting $b=0$, we may regard the de Rham cochain complex $\left(\Omega_{X}^{*}, d\right)$ as a trivial mixed complex $\left(\Omega_{X}^{*}, 0, d\right)[\mathrm{W}, 9.8 .8]$. Since $\left(\Omega_{X}^{*}, 0\right)$ is the product of the sheaves $\Omega_{X}^{p}[-p]$, its hypercohomology is the product of their cohomologies ([W1, A.4]). This yields the following product decomposition

$$
H H_{n}\left(\Omega_{X}^{*}, 0, d\right)=H^{0}\left(X, \Omega_{X}^{n}\right) \times H^{1}\left(X, \Omega_{X}^{n+1}\right) \times \cdots \times H^{p}\left(\Omega_{X}^{n+p}\right) \times \cdots
$$

As $X$ is quasi-compact and quasi-separated (e.g., noetherian), this is a finite decomposition [EGA, III(1.4.12)].

There is a similar decomposition for the cyclic homology of $\left(\Omega_{X}^{*}, 0, d\right)$ and its variants. As a cochain complex, Tot $\mathcal{B}_{* *}\left(\Omega_{X}^{*}, 0, d\right)$ is the product of the translations $\Omega_{X}^{\leq i}[-2 i]$ of its brutal truncations

$$
\Omega_{X}^{\leq i}: \quad 0 \rightarrow \mathcal{O}_{X} \stackrel{d}{\rightarrow} \Omega_{X}^{1} \stackrel{d}{\rightarrow} \cdots \stackrel{d}{\rightarrow} \Omega_{X}^{i} \rightarrow 0 .
$$

As each term of Tot $\left(\mathcal{B}_{* *}\right)$ only involves finitely many of these truncated complexes, $H C_{n}\left(\Omega_{X}^{*}, 0, d\right)$ is $\prod_{i=0}^{\infty} \mathbb{H}^{2 i-n}\left(X, \Omega_{X}^{\leq i}\right)$.

Recall from [Dix, 6.2] that the de Rham cohomology of $X$ over $k$ is defined to be $H_{d R}^{*}(X / k)=\mathbb{H}^{*}\left(X, \Omega_{X}^{*}\right)$. As $\mathbb{H}^{n}\left(X, \Omega_{X}^{*}\right)=\mathbb{H}^{n}\left(X, \Omega_{X}^{\leq i}\right)$ for $i>n$, it follows from 2.1.2 that

$$
H P_{n}\left(\Omega_{X}^{*}, 0, d\right)=\prod^{\infty} H_{d R}^{2 i-n}(X / k)
$$


while $H N_{n}\left(\Omega_{X}^{*}, 0, d\right)$ is the product of the $\mathbb{H}^{2 i-n}\left(X, \Omega_{X}^{\geq i}\right)$.

Hodge-to-de Rham spectral sequence 2.8. The filtration of the cochain complex $\Omega_{X}^{*}$ by the subcomplexes $\Omega_{\bar{X}}^{\geq i}$ gives rise to a spectral sequence

$$
E_{1}^{p q}=H^{q}\left(X, \Omega_{X}^{p}\right) \Rightarrow H_{d R}^{*}(X / k)
$$

called the Hodge-to-de Rham spectral sequence in [Dix, 6.4]. Since this is the same filtration as the column filtration on $\mathcal{B}_{* *}\left(\Omega_{X}^{*}, 0, d\right)$, it follows that Connes' spectral sequence is the product of copies of the Hodge-to-de Rham spectral sequence.

When $X$ is smooth and projective, the $i^{t h}$ piece of the associated Hodge filtration on $H_{d R}^{*}(X / k)$ is the image of the map $\mathbb{H}^{*}\left(X, \Omega_{X}^{\geq i}\right) \rightarrow \mathbb{H}^{*}\left(X, \Omega_{X}^{*}\right)$. The Hodge filtration on the cohomology of $X$ is more complicated in the general case; see $[\mathrm{D}]$.

If $X$ is smooth and projective over a field $k$ of characteristic zero, the spectral sequence degenerates at $E_{1}$ (by Hodge theory on the Kähler manifold $X(\mathbb{C})$ ) to yield the usual "Hodge decomposition":

$$
H_{d R}^{n}(X / k)=\prod_{p+q=n} H^{q}\left(X, \Omega_{X}^{p}\right) .
$$

\section{$\S 3$. The Hodge decomposition of cyclic homology}

We now apply the theory of mixed complexes to study the cyclic homology of $X$. For simplicity, we write $\mathcal{B}_{* *}$ for Connes' $(b, B)$ double complex $\mathcal{B}_{* *}\left(\overline{\mathcal{C}}_{*}\right)(2.6)$; $H C_{*}(X)$ is the hypercohomology of $\mathcal{B}_{* *}$. Recall that the $B$ operator of Connes takes the reduced complex $\overline{\mathcal{C}}_{n}^{(i)}$ to $\overline{\mathcal{C}}_{n+1}^{(i+1)}[\mathrm{L}, 4.6 .7][\mathrm{W}, 9.8 .15]$. Therefore $\mathcal{B}_{* *}$ breaks up as a product (and direct sum) of first quadrant double complexes $\mathcal{B}_{* *}^{(i)}(i \geq 0)$ with

$$
\mathcal{B}_{p q}^{(i)}=\overline{\mathcal{C}}_{q-p}^{(i-p)}
$$

We set $H C_{n}^{(i)}(X)=\mathbb{H}^{-n}\left(X, \operatorname{Tot} \mathcal{B}_{* *}^{(i)}\right)$.

Lemma/Definition 3.0. There is a finite product decomposition for each $n$ :

$$
H C_{n}(X)=H C_{n}^{(0)}(X) \times H C_{n}^{(1)}(X) \times \cdots \times H C_{n}^{(i)}(X) \times \cdots
$$

which we shall call the Hodge decomposition of $H C_{n}(X)$.

For an affine scheme $X=\operatorname{Spec} A$ we have $H C_{n}^{(i)}(X)=H C_{n}^{(i)}(A)$, and this is the usual Hodge decomposition of $H C_{n}(X)=H C_{n}(A)$. Of course, since $\mathcal{B}_{* *}^{(0)}=\mathcal{O}_{X}$, we have $H C_{*}^{(0)}(X)=H H_{*}^{(0)}(X)=H^{-*}\left(X, \mathcal{O}_{X}\right)$, as in (1.1).

Proof. Given Proposition 1.3, the proof of [W1, 2.5] applies to show that for an affine scheme we have $H C_{n}^{(i)}(X)=H C_{n}^{(i)}(A)$. The result now follows from [W1, $0.5]$, as in the proof of 1.3 .

The $S$ map sends $\mathcal{B}_{* *}^{(i)}$ into a translation of $\mathcal{B}_{* *}^{(i-1)}$ up and to the right, which we write as $\mathcal{B}_{* *}^{(i-1)}[-2]$. As in 2.1, we form the tower of hypercohomology complexes 
we define $H P_{n}^{(i)}(X)$ to be the (co)homology of $L_{*}$. We also define $H N_{n}^{(i)}(X)$ to be the (co)homology of the kernel in order to have "SBI" sequences like (2.1.3).

As with cyclic homology, we have decompositions $H P_{n}(X)=\prod H P_{n}^{(i)}(X)$ and $H N_{n}(X)=\prod H N_{n}^{(i)}(X)$, but this time the products are over all integers $i$. Of course, when $X=\operatorname{Spec}(A)$ is affine we see from 3.0 and (2.1.2) that $H P_{n}^{(i)}(X)=$ $H P_{n}^{(i)}(A)$ and $H N_{n}^{(i)}(X)=H N_{n}^{(i)}(A)$.

The affine version of the following result is due to Feigin and Tsygan [FT, 6.5].

Proposition 3.1. If $X$ is locally a complete intersection, then

$$
H C_{n}^{(i)}(X)=0 \quad \text { whenever } i<n / 2 .
$$

Proof. We proceed by induction on $i$, having just checked the case $i=0$. The "SBI" sequence decomposes to yield the exact sequence

$$
H H_{n}^{(i)}(X) \stackrel{I}{\rightarrow} H C_{n}^{(i)}(X) \stackrel{S}{\rightarrow} H C_{n-2}^{(i-1)}(X) .
$$

If $n>2 i$ then the outside terms vanish, by induction and by 1.5. Done.

We now turn to the relation with de Rham cohomology. As in the affine case, there is a morphism of mixed complexes of sheaves $e: \mathcal{C}_{*} \rightarrow\left(\Omega_{X}^{*}, 0, d\right)$ defined by $e\left(a_{0} \otimes \ldots\right)=a_{0} d a_{1} \wedge \cdots \wedge d a_{n} / n$ !. This map sends $\mathcal{C}_{*}^{(i)}$ to $\Omega_{X}^{i}[\mathrm{~W}, 9.8 .12,15]$. By 2.6 , it sends $\mathcal{B}_{* *}^{(i)}$ to $\Omega_{X}^{\leq i}[-2 i]$. Therefore $e$ induces maps

$$
H H_{n}^{(i)}(X) \rightarrow H^{i-n}\left(X, \Omega_{X}^{i}\right) \quad \ldots \quad \text { and } \quad H P_{n}^{(i)}(X) \rightarrow H_{d R}^{2 i-n}(X / k) .
$$

Theorem 3.3. If $X i$ s smooth over $k\left(\right.$ and $\mathbb{Q} \subseteq k$ ) then $H H_{n}^{(i)}(X) \cong H^{i-n}\left(X, \Omega_{X}^{i}\right)$,

$$
\begin{gathered}
H P_{n}^{(i)}(X) \cong H_{d R}^{2 i-n}(X / k), \\
H C_{n}^{(i)}(X) \cong \mathbb{H}^{2 i-n}\left(X, \Omega_{\bar{X}}^{\leq i}\right) \text { and } H N_{n}^{(i)}(X) \cong \mathbb{H}^{2 i-n}\left(X, \Omega_{\bar{X}}^{\geq i}\right) .
\end{gathered}
$$

Proof. Combine 1.4 and (3.2) with 2.5(2), 2.7 and 3.0.

If $X$ is smooth over $k=\mathbb{C}$, Grothendieck's Comparison Theorem [GdR] gives an isomorphism between $H P_{n}^{(i)}(X)$ and the Betti cohomology $H_{\text {top }}^{2 i-n}(X(\mathbb{C}) ; \mathbb{C})$ of the underlying complex manifold $X(\mathbb{C})$.

Example 3.3.1 (Smooth projective curves). If $X$ is a smooth curve then $H C_{n}^{(0)}(X)=H^{-n}\left(X, \mathcal{O}_{X}\right)$ and $H C_{n}^{(1)}(X) \cong H P_{n}^{(1)}(X) \cong H_{d R}^{2-n}(X / k)$. In particular, if $X$ is projective with genus $g$ then $H C_{1}(X)=H C_{1}^{(1)}(X) \cong k^{2 g}$ and $H C_{-1}(X)=H C_{-1}^{(0)}(X) \cong k^{g}$. We will return to this calculation in Examples 3.6 and 4.4.1.

When $X$ is a singular variety over a field of characteristic 0 , the de Rham cohomology $H_{d R}^{*}(X / k)$ is no longer the relevant theory. The useful theory is Hartshorne's "algebraic de Rham cohomology" [H], which we write as $H_{c r i s}^{*}(X / k)$ because (as observed in [Gcr]) it agrees with the crystalline cohomology of the structure sheaf in the crystalline topos for $X$. When the field is $\mathbb{C}$, Hartshorne proved in $[\mathrm{H}, \mathrm{IV} .1 .1]$ that $H_{\text {cris }}^{*}(X / k)$ is isomorphic to the Betti cohomology $H_{\text {top }}^{*}(X(\mathbb{C}) ; \mathbb{C})$ of the underlying topological space $X(\mathbb{C})$. If $X=\operatorname{Spec}(A)$, Feigin and Tsygan proved that $H P_{n}^{(i)}(A) \cong H_{c r i s}^{2 i-n}(A / k)$ (see $\left.[\mathrm{FT}, 6.1][\mathrm{E}][\mathrm{KW}]\right)$. The following theo- 
Comparison Theorem 3.4. When $X$ is a quasi-projective scheme over a field $k$ of characteristic zero, the periodic cyclic homology of $X$ is naturally isomorphic to the crystalline cohomology of $X$ :

$$
H P_{n}^{(i)}(X) \cong H_{c r i s}^{2 i-n}(X / k)
$$

In particular, if $k=\mathbb{C}$ it is isomorphic to the Betti cohomology of $X(\mathbb{C})$ :

$$
H P_{n}^{(i)}(X) \cong H_{\text {top }}^{2 i-n}(X(\mathbb{C}) ; \mathbb{C})
$$

Proof. Using the Comparison Theorem for the Čech cohomology spectral sequences (and [H, II.7.3]), the global version follows from the affine version of Feigin and Tsygan, once we construct a natural map from $H P_{n}^{(i)}(X)$ to $H_{c r i s}^{2 i-n}(X / k)$. This construction will be modelled on the construction of [FT, 6.1.1(1)]. The reader may wish to compare our construction with the analysis in $[\mathrm{Em}][\mathrm{KW}]$.

Take a closed embedding of $X$ in a smooth variety $S$. Each affine open $U=$ $\operatorname{Spec}(R)$ of $S$ contains the affine open $\operatorname{Spec}(A)=X \cap U$ of $X$. By standard techniques we can construct a quasi-isomorphism $\mathcal{P} \rightarrow \mathcal{O}_{X}$, where $\mathcal{P}=\operatorname{Sym}_{S}\left(\oplus V_{i}\right)$ is a free differential graded-commutative $\mathcal{O}_{S}$-algebra on a sequence $V_{1}, V_{2}, \ldots$ of vector bundles on $S$.

The algebra $\Omega_{\mathcal{P}}$ of differential forms may be thought of as a second quadrant homology double complex containing $\Omega_{S}$ on the $x$-axis; Feigin and Tsygan observed in $[\mathrm{FT}, 6.3 .2]$ that, after index shifting, its brutal truncations $\sigma_{p} \Omega_{\mathcal{P}}$ form models for the cyclic homology of each affine open subset of $X$. In [FT, 6.3.3] they extend the filtration $F^{p}$ on $\Omega_{S}^{*}$ to a filtration $J^{p}$ such that the maps $\Omega_{S}^{*} / F^{p} \rightarrow \Omega_{\mathcal{P}} / J^{p}$ are all quasi-isomorphisms. This yields a natural chain of morphisms between towers of complexes whose $p^{\text {th }}$ term is

$$
\operatorname{Tot} \mathcal{B}_{* *}^{(p)}\left(\mathcal{O}_{X}\right)[2 p] \stackrel{\sim}{\leftarrow} \operatorname{Tot} \sigma_{p} \Omega_{\mathcal{P}} \rightarrow \operatorname{Tot} \sigma_{p} \Omega_{\mathcal{P}} / J^{p} \stackrel{\sim}{\longleftarrow} \Omega_{S}^{*} / F^{p} \Omega_{S}^{*}
$$

Now take hypercohomology to get a chain of morphisms between towers of $k$ module complexes. The homotopy limit of the first tower represents the component $H P_{*}^{(0)}(X)$ of the periodic cyclic homology of $X$. The homotopy limit of the last tower is just the hypercohomology of the inverse limit $\hat{\Omega}_{S}^{*}$, which by [H, II.1] represents the algebraic de Rham cohomology $H_{c r i s}^{*}(X / k)=H^{*}\left(\hat{X}, \hat{\Omega}_{S}^{*}\right)$. This yields the desired map from $H P_{n}^{(i)}(X) \cong H P_{n-2 i}^{(0)}(X)$ to $H_{c r i s}^{2 i-n}(X / k)$.

Corollary 3.4.1. (Emmanouil [Em]) If $X$ is quasi-projective over a field of characteristic zero, then every tower $\cdots \stackrel{S}{\longrightarrow} H C_{n+2}(X) \stackrel{S}{\longrightarrow} H C_{n}(X)$ satisfies the MittagLeffler condition and hence $H P_{n}(X)=\varliminf_{p} H C_{n+2 p}(X)$.

Proof. By [H, II.6.1] the $H^{2 i-n}(X / k)=H P_{n}^{(i)}(X)$ are finite-dimensional vector spaces; by [H, II.7.2.1] they are nonzero for only finitely many $i$. Hence each $H P_{n}(X)$ is finite-dimensional. The vector spaces $\varliminf^{1} H C_{n+2 p+1}(X)$ are also finite by Lemma 2.1.2. The result now follows from a theorem of B. Gray (see [Em]). 
Localization of smooth schemes 3.5. Let $X$ be a smooth scheme of dimension $d$ over $k$. Hartshorne [H, II.3] defines the de Rham homology $H_{q}^{d R}(Z)$ of a closed subscheme $Z$ to be hypercohomology with supports $\mathbf{H}_{Z}^{2 d-q}\left(X, \Omega_{X}^{*}\right)$. Thus it is the relative term in the long exact sequence

$\cdots \rightarrow H_{q}^{d R}(Z) \rightarrow H_{d R}^{2 d-q}(X) \rightarrow H_{d R}^{2 d-q}(X-Z) \rightarrow H_{q-1}^{d R}(Z) \rightarrow H_{d R}^{2 d-q+1}(X) \rightarrow \cdots$.

Using 3.3, it is also the relative term in the periodic cyclic homology sequence:

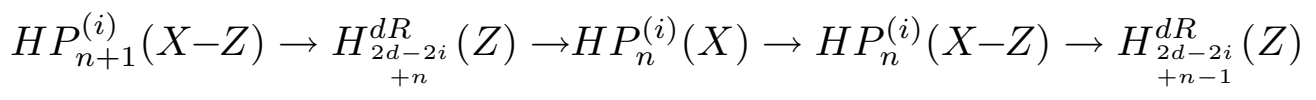

If in addition $Z$ is also smooth, of codimension $c$ (and hence dimension $d-c$ ), then the above sequence simplifies into the more attractive sequence:

$$
H P_{n+1}^{(i)}(X-Z) \rightarrow H P_{n}^{(i-c)}(Z) \rightarrow H P_{n}^{(i)}(X) \rightarrow H P_{n}^{(i)}(X-Z) \rightarrow H P_{n-1}^{(i-c)}(Z)
$$

Example 3.6 (Smooth affine curves). Let $A$ be the coordinate ring of a smooth affine curve $X$ obtained from a smooth projective curve $\bar{X}$ by removing $n \geq 1$ points, whose corresponding residue fields are $k_{1}, \ldots, k_{n}$. Suppose for simplicity that $\bar{X}$ is connected with genus $g$, so that $H C_{1}(\bar{X})=H P_{1}(\bar{X})=k^{2 g}$ by 3.3.1. Since $H C_{1}(A)=H P_{1}(A)$ and the maps $H_{0}^{d R}\left(k_{i}\right) \rightarrow H_{0}^{d R}(\bar{X})$ are the trace maps $k_{i} \rightarrow k$ by [H, II.3.1], the Localization Sequence (3.5.2) (or [H, II.3.3]) translates into the exact sequence

$$
0 \rightarrow k^{2 g} \rightarrow H C_{1}(A) \rightarrow \coprod_{i=1}^{n} k_{i} \stackrel{\text { trace }}{\longrightarrow} k \rightarrow 0 .
$$

The image of the first map contains the $g$ global holomorphic differentials on $\bar{X}$, considered as elements of $H C_{1}(A)=\Omega_{A} / d A$. In particular, if $k=\mathbb{C}$ (or if $k_{i}=k$ for all $i$ ) then the dimension of $H C_{1}(A)$ is $2 g+n-1$.

As an exercise, the reader might want to check this for the hyperelliptic plane curve $A=\mathbb{C}[x, y] /\left(y^{2}=f(x)\right), f$ a degree $d$ polynomial with distinct roots. In this case the genus is $\left[\frac{d-1}{2}\right]$, and there are either 1 or 2 points on $\bar{X}-X$. Thus $\operatorname{dim} H C_{1}(A)=d-1$.

To see the effect of the trace, consider the coordinate ring of the circle $A=$ $\mathbb{R}[x, y] /\left(x^{2}+y^{2}=1\right)$. Since there is 1 point at infinity with residue field $\mathbb{C}$, and $g=0$, this yields $H C_{1}(A)=\mathbb{R}(1)$, the kernel of the trace $\mathbb{C} \rightarrow \mathbb{R}$.

Localization Theorem 3.7. Let $X$ be a d-dimensional quasi-projective scheme $X$, and suppose that $Z$ is a closed subscheme disjoint from the singular locus of $X$. Then the relative term in the localization sequence for periodic cyclic homology is Hartshorne's algebraic de Rham homology, with a shift of $s=2 d-2 i$. That is, the sequence (3.5.1) is exact:

$$
\cdots H P_{n+1}^{(i)}(X-Z) \rightarrow H_{s+n}^{d R}(Z) \rightarrow H P_{n}^{(i)}(X) \rightarrow H P_{n}^{(i)}(X-Z) \rightarrow H_{s+n-1}^{d R}(Z) \cdots
$$

If in addition $Z$ is smooth, the relative term is also the periodic cyclic homology of $Z$, with the Hodge index shifted by the codimension $c$ of $Z$ in $X$. That is, the sequence (3.5.2) is exact: 
Proof. Set $Y=\operatorname{Sing}(X)$. It suffices by 3.5 to show that the relative terms agree for the maps $H_{c r i s}^{*}(X / k) \rightarrow H_{c r i s}^{*}(X-Z / k)$ and $H_{c r i s}^{*}(X-Y / k) \rightarrow H_{c r i s}^{*}(X-Y-Z / k)$. This follows from the Mayer-Vietoris sequence of [H, II.7.3]:

$\cdots H_{\text {cris }}^{*}(X) \rightarrow H_{\text {cris }}^{*}(X-Y) \oplus H_{\text {cris }}^{*}(X-Z) \rightarrow H_{c r i s}^{*}(X-Y-Z) \rightarrow H_{c r i s}^{*}(X) \cdots$.

More directly, one could embed $X$ in a smooth variety $S$ and set $U=S-Y$. The two relative terms in question are the hypercohomology groups with supports: $\mathbb{H}_{Z}^{*}\left(\hat{S}, \hat{\Omega}_{S}^{*}\right)$ and $\mathbb{H}_{Z}^{*}\left(\hat{U}, \hat{\Omega}_{U}^{*}\right)$. By excision, these are isomorphic, so we are done.

Remark. If $Z$ is projective but not smooth, then by [H, II.5.1] we know that $H_{q}^{d R}(Z)$ and $H_{c r i s}^{q}(Z / k)$ are dual vector spaces. We leave the transformation of the localization sequences of 3.7 in the general case to the reader.

If $Z$ is not even projective, then by [H, IV.1.2] the de Rham homology of $Z$ over $\mathbb{C}$ is the same as the Borel-Moore homology of the locally compact topological space $Z(\mathbb{C})$; this is the same as homology with locally compact supports because the coefficients are taken in $\mathbb{C}$.

Chern characters 3.8 We conclude this section with a discussion of Chern characters. There is a Chern character ch: $K_{0}(X) \rightarrow H N_{0}(X)$ for schemes, generalizing the usual affine Chern character $c h: K_{0}(A) \rightarrow H N_{0}(A)$ [L, 8.3.8]. It is described in [WG, 4.4], and is obtained by sheafifying the higher Chern character $[\mathrm{L}, 11.4 .2]$ and taking hypercohomology as in $[\mathrm{T}]$.

The composition of $c h$ with $H N_{0}(X) \rightarrow H H_{0}(X)$ yields a natural generalization to schemes of the classical Hattori-Stallings trace map $K_{0}(A) \rightarrow H H_{0}(A)$. We shall call it the Dennis trace map, since it is obtained by sheafifying the Dennis trace map (for higher $K$-theory) and taking hypercohomology as in [T]. We will see below (in 4.5 ) that for $X$ smooth and projective the Dennis trace map completely determines the classical Chern classes of $X$.

The composition of $c h$ with $H N_{0}(X) \rightarrow H P_{0}(X)$ is a generalization to schemes of the Connes-Karoubi Chern character. Note that $H P_{0}(X)=\prod H_{c r i s}^{2 i}(X / k)$ by the Comparison Theorem 3.4.

Proposition 3.8.1. For quasiprojective schemes, the Connes-Karoubi Chern character $K_{0}(X) \stackrel{\text { ch }}{\longrightarrow} H P_{0}(X)=\prod H_{c r i s}^{2 i}(X / k)$ agrees with the Chern character associated to the Chern classes constructed by Hartshorne in [H, II.7.9].

Proof. If $X$ is smooth, this is [W2, Thm.1]. The general case follows from Jouanalou's device and [W2, Lemme 6].

\section{$\S 4$. Hodge filtration for Smooth Projective Varieties}

If $X$ is a projective variety over a field $k$ of characteristic 0 , there is a connection between the filtration on $H^{m}(X / k)$ defined by the system of maps

$$
H^{m}(X / k) \rightarrow \cdots \rightarrow H C_{2 i-m}^{(i)}(X) \rightarrow H C_{2 i-m-2}^{(i-1)}(X) \rightarrow \cdots
$$

defined by the comparison theorems in $\S 3$, and the Hodge filtration $H^{m}=F^{0} \supseteq$ $F^{1} \supseteq \cdots$ on $H_{\text {top }}^{m}(X(\mathbb{C}), \mathbb{C})$ defined by Deligne in $[\mathrm{D}]$. This connection is clearest for smooth $X$, where $H^{m}(X / k)$ is de Rham cohomology, and is given by the following 
Proposition 4.1 (Smooth projective varieties). If $X$ is a smooth projective variety over a field $k$ (and char $(k)=0$ ) then:

a) The $i^{\text {th }}$ part $F^{i} H^{m}(X / k)$ of the Hodge filtration on $H_{d R}^{m}(X / k)$ is isomorphic to $H N_{2 i-m}^{(i)}(X)$, via the map $I: H N_{2 i-m}^{(i)}(X) \rightarrow H P_{2 i-m}^{(i)}(X) \cong H_{d R}^{m}(X / k)$.

b) The "SBI" sequences break up into short exact sequences:

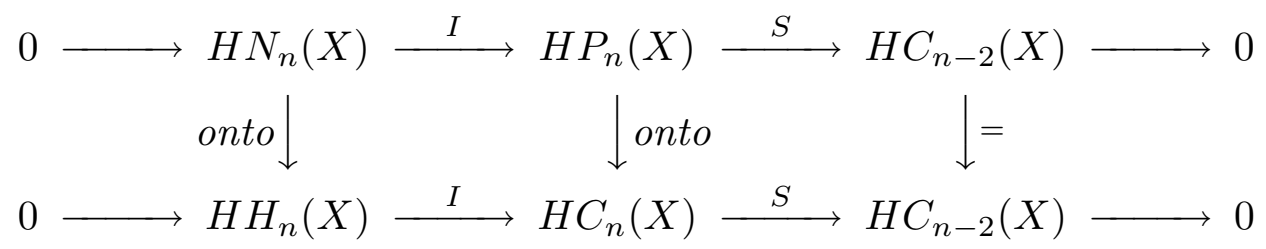

c) $H P_{n}^{(i)}(X)$ is the product of the $H^{q}\left(X, \Omega_{X}^{p}\right), p+q=2 i-n$, and

$$
H N_{n}^{(i)}(X)=\prod_{\substack{p+q=2 i-n \\ p \geq i}} H^{q}\left(X, \Omega_{X}^{p}\right), \quad H C_{n}^{(i)}(X)=\prod_{\substack{p+q=2 i-n \\ p \leq i}} H^{q}\left(X, \Omega_{X}^{p}\right)
$$

Proof. In this case the Hodge-to-de Rham spectral sequence 2.8 degenerates. Thus $H P_{n}^{(i)}(X), H C_{n}^{(i)}(X)$ and $H N_{n}^{(i)}(X)$ are direct products, as described in c). Part b) follows by taking the product over the Hodge components. Assertion a) about the $i^{t h}$ part of the Hodge filtration of $H_{d R}^{m}(X / k)$ follows from this and 2.8.

Remark 4.2. When $X$ is a smooth affine variety over $\mathbb{C}$ none of 4.1 holds, even though we still have $H_{d R}^{*}(X / \mathbb{C}) \cong H_{\text {top }}^{*}(X(\mathbb{C}) ; \mathbb{C})$ by $[\mathrm{GdR}]$. It is well-known that the "SBI" sequence breaks up differently, with $H C_{n}^{(n)}(X) \cong \Omega_{A}^{n} / d \Omega_{A}^{n-1}$. Moreover the Hodge filtration (see $[\mathrm{D}, 3.2 .2]$ ) is more complicated and has less of a relation to cyclic homology. Consider for example a smooth affine curve $X=\operatorname{Spec} A$ of genus $g \neq 0$ over $\mathbb{C}$. The Hodge filtration on $H_{d R}^{1}(X / \mathbb{C})$ is nontrivial by $[\mathrm{D}, 8.2 .5]$ yet, as we see from Example 3.6, the map from $H N_{1}^{(1)}(X)=\Omega_{A}$ to $H P_{1}^{(1)}(X)=$ $H_{d R}^{1}(X) \cong \Omega_{A} / d A$ is onto with kernel $A / \mathbb{C}$. In this case, the Hodge-to-de Rham spectral sequence 2.8 degenerates only at $E_{2}$, unlike the spectral sequence for the Hodge filtration, which degenerates at $E_{1}$ by [D, 3.2.13].

The Hodge Diamond 4.3. Let $X$ be a smooth projective variety. It is traditional to display the Hodge numbers $h^{p q}=\operatorname{dim} H^{q}\left(X, \Omega_{X}^{p}\right)$ of $X$ in the shape of a diamond, so that $n=p-q$ is constant in each column and $m=p+q$ is constant in each row, with the $p=(m+n) / 2$ increasing from left to right as well as down to up. (See examples 4.4 below.) In this format, the $m^{\text {th }}$ row displays the classical Hodge decomposition of $H^{m}(X(\mathbb{C}) ; \mathbb{C})$, as well as the Hodge filtration; $\operatorname{dim} F^{i} H^{m}$ is the sum of the terms in the $m^{t h}$ row which lie in columns $n \geq(2 i-m)$.

The Hodge decomposition of $H H_{n}(X)$ is given by the $n^{t h}$ column of the Hodge Diamond. Indeed, the entry in row $m=2 i-n$ of the $n^{t h}$ column is $h^{i-n, n}$, which by 1.4 is the dimension of $H H_{n}^{(i)}(X)$. Serre Duality implies that $H H_{+n}(X)$ and $H H_{-n}(X)$ are dual vector spaces.

The rows of the Hodge diamond describe the Hodge decomposition of $H C_{n}(X)$ as follows. Fix $n$ and $i$, and set $m=2 i-n$. By Proposition 4.1, the sum of the terms in the $m^{t h}$ row to the left and including the $n^{t h}$ column gives the dimension of 
Hodge decomposition of $H C_{n}(X)$ is given by the rows of this truncated diamond. Of course, the $S$ maps correspond to truncation, and the sum of all the terms in the $m^{t h}$ row is the dimension of $H P_{n}^{(i)}(X) \cong H^{m}(X(\mathbb{C}) ; \mathbb{C})$.

Example 4.4.1 (Smooth projective curves). Let $X$ be a smooth projective curve of genus $g$ over a field $k$ of characteristic 0 . From the Hodge diamond

\begin{tabular}{|ccccc|}
\hline & 1 & & $H^{2}(X ; \mathbb{C})$ \\
& & $g$ & $H^{1}(X ; \mathbb{C})$ \\
& 1 & & $H^{0}(X ; \mathbb{C})$ \\
$H H_{-1}$ & $H H_{0}$ & $H H_{1}$ & \\
\hline
\end{tabular}

and 4.1 we see that $H H_{+1}(X) \cong H H_{-1}(X) \cong k^{g}, H P_{1}(X)=H C_{1}(X)=k^{2 g}$ but $H C_{-1}(X)=k^{g}$, and that

$$
H P_{0}(X)=H C_{0}(X) \cong H H_{0}(X) \cong k \oplus k, \text { but } H C_{-2}(X) \cong k .
$$

Example 4.4.2 (Smooth projective surfaces). Consider a smooth projective surface $X$ of genus $g$ and irregularity $q$. The Hodge diamond looks like this:

\begin{tabular}{|ccccccc}
\hline & & $h^{22}$ & & & $H^{4}(X ; \mathbb{C})$ \\
$h^{02}$ & $h^{12}$ & & $h^{21}$ & & $H^{3}(X ; \mathbb{C})$ \\
& & $h^{11}$ & & $h^{20}$ & $H^{2}(X ; \mathbb{C})$ \\
& & $h^{00}$ & $h^{10}$ & & $H^{1}(X ; \mathbb{C})$ \\
& & & & $H^{0}(X ; \mathbb{C})$ \\
$H H_{-2}$ & $H H_{-1}$ & $H H_{0}$ & $H H_{1}$ & $H H_{2}$ & \\
\hline
\end{tabular}

Since $g=h^{02}=h^{20}$ and $q=h^{01}=h^{12}=h^{10}=h^{21}$ we have

$$
H H_{2}(X) \cong H H_{-2}(X) \cong k^{g}, \quad H H_{1}(X) \cong H H_{-1}(X) \cong k^{2 q},
$$

and $H H_{0}(X)=k \oplus H H_{0}^{(1)}(X) \oplus k$ with $H H_{0}^{(1)}(X)=H^{1}\left(X, \Omega_{X}\right)=k^{h^{11}}$. The classical group of divisors modulo numerical equivalence is free abelian of rank $h^{11}$ and injects into $H H_{0}^{(1)}(X)$ under the Chern class $c_{1}$. The sequence of $S$-maps

$$
H^{2}(X ; \mathbb{C})=H C_{2}^{(2)}(X) \rightarrow H C_{0}^{(1)}(X) \rightarrow H C_{-2}^{(0)}(X)=k^{g}
$$

give the Hodge filtration of $H^{2}(X ; \mathbb{C})$.

Proposition 4.5. When $X$ is a smooth projective variety, the classical Chern character $K_{0}(X) \rightarrow \prod H_{d R}^{2 i}(X)$ factors through the Dennis trace map

$$
K_{0}(X) \rightarrow H H_{0}(X)=\prod_{i=0}^{\operatorname{dim} X} H^{i}\left(X, \Omega_{X}^{i}\right) .
$$

Proof. Since the classical Chern classes

$$
c_{i}: K_{0}(X) \rightarrow H_{d R}^{2 i}(X)
$$

land in the submodules $H H_{0}^{(i)}(X)=H^{i}\left(X, \Omega_{X}^{i}\right)$, it follows from 3.8.1 that the image of ch lies in the submodule $H H_{0}(X)=\prod H^{i}\left(X, \Omega_{X}^{i}\right)$ of $H N_{0}(X)$. Since the composition of $c h$ with $H N_{0}(X) \rightarrow H H_{0}(X)$ is the Dennis trace map, we are done. 


\section{$\S 5$ Hodge Structure for Singular Varieties}

Let $X$ be a singular projective variety over $\mathbb{C}$. The Hodge filtration $F^{i} H^{m}$ on $H^{m}(X, \mathbb{C})=H_{\text {top }}^{m}(X(\mathbb{C}), \mathbb{C})$ was defined by Deligne in $[\mathrm{D}, 8.2 .2]$ as a part of the mixed Hodge structure on $H^{m}(X ; \mathbb{Z})$. To define it, we choose a smooth proper hypercovering $\pi: X_{\bullet} \rightarrow X$, as in [D, 6.2.8], and observe that $H^{m}(X, \mathbb{C})=$ $\mathbb{H}^{m}\left(X_{\bullet} ; \Omega_{X_{\bullet}}^{*}\right)$ by $[\mathrm{D}, 5.3 .5(\mathrm{~V})]$. The Hodge filtration is given by the hypercohomology of the brutal truncations $\Omega_{X}^{\geq i}$ of the simplicial cochain complex $\Omega_{X}^{*}$. $[\mathrm{D}, 8.1 .8]$. We remark that the mixed Hodge structure on $H^{m}(X, \mathbb{Z})$ is defined in $[\mathrm{D}, 8.1 .12$, 8.1.19 and 8.2.2], using the cohomological mixed Hodge structure on the constant sheaf $\mathbb{Z}$.

Theorem 5.1. Let $X$ be a projective variety over $\mathbb{C}$, and fix $m, n$ with $m+n=2 i$.

a) The image of $H N_{n}^{(i)}(X) \rightarrow H P_{n}^{(i)}(X) \cong H^{m}(X, \mathbb{C})$ is contained in $F^{i} H^{m}(X, \mathbb{C})$.

b) $H C_{n}^{(i)}(X)$ surjects onto $H^{m}(X, \mathbb{C}) / F^{i+1} H^{m}$.

c) If $i \geq m$ then $H^{m}(X, \mathbb{C})=H P_{n}^{(i)}(X)$ injects into $H C_{n}^{(i)}(X)$.

Proof. The mixed complex $\overline{\mathcal{C}}_{*}^{(i)}\left(X_{\bullet}\right)$ is isomorphic to the de Rham mixed complex $\Omega_{X}^{*}$ of Example 2.7, by the maps of $(3.2)$. Hence Tot $\mathcal{B}_{* *}^{(i)}\left(X_{\bullet}\right)$ is quasi-isomorphic to the truncated de Rham complex $\Omega_{X}^{\leq i}[-2 i]$ on $X_{\bullet}$. Hence there are natural maps

$$
\begin{aligned}
H N_{n}^{(i)}(X) & \rightarrow \mathbb{H}^{m}\left(X, \Omega_{X}^{\geq i}\right) \rightarrow \mathbb{H}\left(X_{\bullet}, \Omega_{X}^{\geq i}\right)=F^{i} H^{m}(X, \mathbb{C}), \\
H C_{n}^{(i)}(X) & =\mathbb{H}^{-n}\left(X, \operatorname{Tot} \mathcal{B}_{* *}^{(i)} X\right) \rightarrow \mathbb{H}^{-n}\left(X_{\bullet}, \operatorname{Tot} \mathcal{B}_{* *}^{(i)}\left(X_{\bullet}\right)\right. \\
& =\mathbb{H}^{2 i-n}\left(X_{\bullet}, \Omega_{X_{\bullet}}^{\leq i}\right)=H^{m}(X, \mathbb{C}) / F^{i+1} H^{m} .
\end{aligned}
$$

Part (c) follows from (b) and the vanishing of $F^{m+1} H^{m}(X, \mathbb{C})[\mathrm{D}, 8.2 .4(\mathrm{iii})]$.

Corollary 5.2. Let $X$ be a connected singular projective variety with c irreducible components. If $\operatorname{dim} X=d$ then $H P_{0}^{(d)}(X)=\mathbb{C}^{c}$ injects into $H C_{0}^{(d)}(X)$.

Proof. The Hodge filtration on $H^{2 d}(X, \mathbb{C})$ has $F^{d+1} H^{2 d}=0, F^{d} H^{2 d}=H^{2 d}=\mathbb{C}^{c}$.

Definition 5.3. The "SBI" filtration $H^{m}=G^{0} \supseteq G^{1} \supseteq \cdots$ on $H^{m}=H^{m}(X, \mathbb{C})$ is defined by: $G^{i} H^{m}$ is the image of $H N_{n}^{(i)}(X) \rightarrow H P_{n}^{(i)}(X) \cong H^{m}(X, \mathbb{C}), m+n=2 i$. Thus $H^{m} / G^{i+1} H^{m}$ is the image of $H^{m}$ in $H C_{n}^{(i)}(X)$.

By 5.1, the "SBI" filtration is always contained in the Hodge filtration of a projective variety, and $G^{m+1} H^{m}=0$. By 4.2, the "SBI" filtration coincides with the Hodge filtration for smooth projective varieties. The "SBI" and Hodge filtrations agree on $H^{0}$, where they are trivial. The "SBI" filtration also coincides with the Hodge filtration on $H^{2 \operatorname{dim} X}$ by 5.2. This raises the following question.

Question 5.3.1. When does the "SBI" filtration coincide with the Hodge filtration on a projective variety $X$ ? Equivalently, when is the image of $H^{m}(X, \mathbb{C})$ in $H C_{n}^{(i)}(X)$ exactly $H^{m} / F^{i+1} H^{m}$ ? The next example shows that the two filtrations are not always equal.

Example 5.4. ( $H^{1}$ of Projective Curves) Let $X$ be a connected, reduced singular projective curve. Then $H^{1}$ injects into $H C_{1}^{(1)}(X)$ by $5.1(\mathrm{c})$, so $G^{2} H^{1}=F^{2} H^{1}=0$. We claim that the inclusion $G^{1} H^{1} \subseteq F^{1} H^{1}$ is not always equality, i.e., that the 
For this, we need to analyze the mixed Hodge structure on $H^{1}(X, \mathbb{C})$. If $X$ has $c$ irreducible components then so does the normalization $\tilde{X}$ of $X$; let $g_{1}, \ldots, g_{c}$ be the genera of these components. The singular locus $Y$ of $X$ is finite, and so is its inverse image $\tilde{Y}=Y \times_{X} \tilde{X}$ in $\tilde{X}$. By [H, II.4.4] and 4.4.1, $H P_{1}(X)=H^{1}(X, \mathbb{C})$ fits into an extension

$$
0 \rightarrow W_{0} \rightarrow H P_{1}(X) \rightarrow \oplus \mathbb{C}^{2 g_{i}} \rightarrow 0, \quad \operatorname{dim} W_{0}=1+|\tilde{Y}|-|Y|-c .
$$

By $[\mathrm{D}, 8.2 .5], W_{0}$ is the weight 0 subspace for the mixed Hodge structure of $H^{1}(X, \mathbb{C})$. In fact, $W_{0}$ is the subspace $H^{00}$ and its dimension is the Hodge number $h^{00}[\mathrm{D}, 8.2 .4]$. In this case the Hodge filtration has $F^{1} H^{1}=\oplus \mathbb{C}^{g_{i}}$ by [D, 8.2.5].

Since $X$ is reduced and proper, $H C_{0}^{(0)}(X)=H^{0}\left(X, \mathcal{O}_{X}\right)=\mathbb{C}$. The "SBI" sequence

$$
H C_{0}^{(0)}(X) \stackrel{B}{\longrightarrow} H H_{1}^{(1)}(X) \rightarrow H C_{1}^{(1)}(X) \stackrel{S}{\longrightarrow} H C_{-1}^{(0)}(X) \stackrel{B}{\longrightarrow} H H_{0}^{(1)}(X)
$$

translates via Example 1.6 into the exact sequence

$$
\mathbb{C} \stackrel{0}{\rightarrow} H^{0}\left(X, \Omega_{X}\right) \rightarrow H C_{1}^{(1)}(X) \stackrel{S}{\rightarrow} H^{1}\left(X, \mathcal{O}_{X}\right) \stackrel{d}{\rightarrow} H^{1}\left(X, \Omega_{X}\right) .
$$

Comparing with 4.4.1, we see that the image of $F^{1} H^{1}$ in $H^{1} / G^{1} \subseteq H^{1}\left(X, \mathcal{O}_{X}\right)$ is isomorphic to the cokernel of $H^{0}\left(X, \Omega_{X}\right) \rightarrow H^{0}\left(\tilde{X}, \Omega_{\tilde{X}}\right)$.

Let $X$ be the projective cusp obtained from the elliptic curve $y^{2} z=x^{3}-x z^{2}$ by glueing the origin $(0: 0: 1)$ to itself. Then $Y=\operatorname{Spec} \mathbb{C}$ and $\tilde{Y}=\operatorname{Spec} \mathbb{C}[\varepsilon]$, and the canonical 1-form $\omega=d y$ generating $H^{0}\left(\tilde{X}, \Omega_{\tilde{X}}\right)=\mathbb{C}$ cannot come from $H^{0}\left(X, \Omega_{X}\right)$ because it maps to $d \varepsilon \neq 0$ in $\Omega_{\tilde{Y} / Y}$. For this curve $X$ we therefore have $G^{1} H^{1}=0$ but $F^{1} H^{1} \cong \mathbb{C}$.

Example 5.5. Now let $U=\operatorname{Spec}(A)$ be a singular affine curve, obtained from a (singular) projective curve $X$ by removing $n$ smooth points. The cyclic homology of $X$ is given by the previous example. Suppose for simplicity that $U$ is connected and has $c$ irreducible components. Since $H^{m}(U)=0$ for $m \geq 2$, the localization sequence of 3.7 and 3.4 yields $H P_{0}(A)=H P_{0}^{(0)}(A)=k$, and fits $H P_{1}(A)=$ $H P_{1}^{(1)}(A)$ into an exact sequence

$$
0 \rightarrow H P_{1}(X) \rightarrow H P_{1}(A) \rightarrow k^{n} \stackrel{\delta}{\rightarrow} k^{c} \rightarrow 0,
$$

where $\delta$ is the incidence matrix (of points and irreducible components). Since $\delta$ is onto, $H P_{1}(A) \cong H P_{1}(X) \oplus k^{n-c}$. Thus $H P_{*}(A)$ completely determines $H P_{*}(X)$.

Every vector space $H C_{n}(A)$ is finite-dimensional (when $A$ is a curve). This is a consequence of Example 3.6, the analytic isomorphisms sequence of [GRW, A.1], and the calculations of [GRW]. The method of [GRW, §6] allows us to compute the cyclic homology of all seminormal affine curves, and in principle of all curves.

A lower bound for $H C_{2 i-1}(A)$ is given by $H P_{1}(A)=H P_{1}^{(1)}(A)$, because the map $H P_{1}(A) \rightarrow H C_{i}^{(2 i-1)}(A) \rightarrow H C_{1}(A)$ is an injection. (This theorem is proven in [Em, Remark 4] [KW, 5.4]; it follows from the main theorem of [BH] and the fact that $H C_{1}(A)$ contains $H_{d R}^{1}(A / \mathbb{C})$.) And the calculations of $[\mathrm{GRW}]$ show that 
Remark 5.6. For curves, the subgroup $H^{1}(X / k)=H P_{1}(X)$ is the obstruction to naïve étale descent for periodic cyclic homology in the sense of [WG]. This is illustrated by the coordinate ring $A$ of a line and a parabola in the plane. In this case $n=c=2$ and we have $H P_{1}(A)=k$, with $H C_{2 p+1}(A)=H P_{1}(A) \oplus k^{2}$ for all $p \geq 0$. (This example was discussed in [WG, 3.1].) 


\section{REFERENCES}

[BH] T. Bloom and M. Herrerra, De Rham cohomology of an analytic space, Invent. Math. 7 (1969), 275-296.

[BK] A. Bousfield and D. Kan, Homotopy Limits, Completions, and Localization, Lecture Notes in Math., vol. 304, Springer-Verlag, 1972.

[BN] M. Bökstedt and A. Neemon, Homotopy limits in triangulated categories, Compositio Math. 86 (1993), 209-234.

[D] P. Deligne, Théorie de Hodge II et III, Publ. Math. IHES 40 (1972), 5-57; 44 (1974), $5-78$.

[Dix] A. Grothendieck, Classes de Chern et répresentations linéaires des groups discrets, Dix exposés sur la Cohomologie des Schémas, North-Holland, Amsterdam, 1968, pp. 215-305.

[EGA] A. Grothendieck and J. Dieudonné, Eléments de Géométrie Algébrique III et IV (Première Parties), Publ. Math. IHES 11 (1961); 20 (1964).

[Em] I. Emmanouil, Cyclic homology and the de Rham cohomology of commutative algebras, C.R. Acad. Sci. (Paris) 318 (1994), 413-417.

[FT] B. Feigin and B. Tsygan, Additive K-theory, Lecture Notes in Math., vol. 1289, SpringerVerlag, 1987, pp. 97-209.

[Gcr] A. Grothendieck, Crystals and the de Rham cohomology of algebraic varieties, Dix Exposés sur la Cohomologie des Schémas, North Holland, Amsterdam, 1968, pp. 306-358.

[GdR] A. Grothendieck, On the de Rham cohomology of algebraic varieties, Publ. Math. IHES 29 (1966), 351-359.

[GH] P. Griffiths and J. Harris, Principles of Algebraic Geometry, Wiley-Interscience, 1978.

[Go] T. Goodwillie, Cyclic homology, derivations and the free loop space, Topology 24 (1985), $187-215$.

[GRW] S. Geller, L. Reid and C. Weibel, The cyclic homology and K-theory of curves, J. reine angew. Math. 393 (1989), 39-90.

[GS] M. Gerstenhaber and S.D. Schack, A Hodge-type decomposition for commutative algebra homology, J. Pure Appl. Alg. 48 (1987), 229-247.

[H] R. Hartshorne, On the de Rham cohomology of algebraic varieties, Publ. Math IHES 45 (1976), 5-99.

[KW] C. Kassel and C. Weibel, Excision for periodic cyclic homology of commutative algebras, In preparation.

[L] J.-L. Loday, Cyclic Homology, Springer-Verlag, 1992.

[Q] D. Quillen, On the (co)-homology of commutative rings, Proc. Symp. Pure Math., vol. 17, 1970, pp. 65-87.

[RD] R. Hartshorne, Residues and Duality, Lecture Notes in Math., vol. 20, Springer-Verlag, 1966.

[Ro] M. Ronco, On the Hochschild homology decompositions, Commun. in Algebra 21 (1993), 4699-4712.

[Sp] N. Spaltenstein, Resolutions of unbounded complexes, Compositio Math. 65 (1988), 121154.

[T] R. Thomason, Algebraic K-theory and etale cohomology, Ann. Scient. Éc. Norm. Sup. 18, $4^{e}$ série (1985), 437-552.

[V] M. Vigué-Poirrier, Décompositions de l'homologie cyclique des algèbres différentielles graduées commutatives, K-theory 4 (1991), 399-410.

[W] C. Weibel, An introduction to homological algebra, Cambridge University Press, 1994.

[W1] C. Weibel, Cyclic homology for schemes, preprint, Proc. AMS (1994) (to appear).

[W2] C. Weibel, Le caractère de Chern en homologie cyclique périodique, C.R. Acad. Sci. (Paris) 317 (1993), 867-871.

[WG] C. Weibel and S. Geller, Étale descent for Hochschild and cyclic homology, Comment. Math. Helv. 66 (1991), 368-388.

Math. Dept., Rutgers University, New Brunswick, N.J. 08903 USA

E-mail address: weibel@math.rutgers.edu 